Revue belge de géographie

\title{
Thèses de doctorat et mémoires de licence en géographie/Doctoraats- en licentie- en masterverhandelingen geografie (1999-2003)
}

\section{(2) OpenEdition}

\section{Journals}

Édition électronique

URL : http://journals.openedition.org/belgeo/16257

DOI : $10.4000 /$ belgeo.16257

ISSN : 2294-9135

Éditeur :

National Committee of Geography of Belgium, Société Royale Belge de Géographie

Édition imprimée

Date de publication : 30 juin 2003

Pagination : 195-222

ISSN : 1377-2368

Référence électronique

"Thèses de doctorat et mémoires de licence en géographie/Doctoraats- en licentie- en

masterverhandelingen geografie (1999-2003) », Belgeo [En ligne], 2 | 2003, mis en ligne le 30 juillet 2003, consulté le 30 avril 2019. URL : http://journals.openedition.org/belgeo/16257 ; DOI : 10.4000/ belgeo.16257

Ce document a été généré automatiquement le 30 avril 2019.

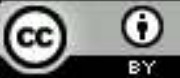

Belgeo est mis à disposition selon les termes de la licence Creative Commons Attribution 4.0 International. 


\section{Thèses de doctorat et mémoires de} licence en géographie/Doctoraats- en licentie- en masterverhandelingen geografie (1999-2003) ${ }^{1}$

\section{Thèses de doctorat - Doctoraatsverhandelingen}

\section{- Katholieke Universiteit Leuven}

1999

BEUSELINCK Laurent: Sediment deposition by overland flow. An experimental and modelling approach.

3 CABUS Peter: De geografie van de ondernemingsstrategie. De toelevering aan de Belgische auto-industrie als onderlegger.

2000

5 TAKKEN Ingrid: Effects of soil roughness on overland flow and erosion.

6 VANDEKERCKHOVE Liesbeth: Gully initiation and development in Mediterranean envirmonments.

7 VERSTRAETEN Gert: Modderoverlast, sedimentatie in wachtbekkens en begroting van de sedimentexport naar waterlopen in Midden-België.

$8 \quad 2001$

9 NACHTERGAELE Jeroen: A spatial and temporal analysis of the characteristics, importance and prediction of ephemeral gully of erosion.

10 NYSSEN Jan: Erosion processes and soil conservation in a tropical mountain catchment under threat of anthropogenic desertification. A case study from Northern Etiopia.

11 STEEGEN Anna: Sediment deposition in and export from small agricultural catchments. 
VAN MUYSEN Wouter: Tillage translocation and tillage erosion: an experimental approach.

VAN ROMPAEY Anton: Geomorphic and land use change modelling at a regional scale.

2002

PELEMAN Katleen: De rol van de buurt: De maatschappelijke participatie van Marokkaanse vrouwen in een ruimtelijk perspectief.

VANACKER Veerle: Geomorphic response to human-indued environmental change in tropical mountain areas.

2003

VAN OOST Kristof: Spatial modelling of soil redistribution processes in agricultural landscapes.

\section{- Rijksuniversiteit Gent}

\section{9}

BASTIDAS de Calderon: Environmental fragility and vulnerability assessment of Amazonian landscapes and ecosystems in the Middle Orinoco river basin, Venezuela.

\section{0}

HOUTHAEVE Rik: Ruimtelijke planvorming in actie: conceptie en toepassing van een «collaboratieve planningspraktijk in Vlaanderen». Met illustratief cases-onderzoek. Kritische succesfactoren voor de «werking» van een ruimtelijk planningsstelsel.

\section{1}

DEJONGHE Trudo: De noodzaak aan exogeen opgelegde economisch-geografische principes bij het professionaliseren van het door endogene factoren ontstane wereldsportstelsel. Gevalstudie: Het lokalisatievraagstuk van het topvoetbal in België.

VANWESENBEECK Veerle: Geomorfologisch en sedimentologisch onderzoek op meso- en microschaal in de maritieme Schelde tussen Antwerpen en de Belgisch-Nederlandse grens.

2002

BRONDEEL Marijke: De invloed van de atmosferische belasting op coördinaten bepaald door de ruimtegeodesie, meer in het bijzonder met het GPS systeem.

DE WACHTER Astrid: De toepassing van wereldsysteem-analyse op geografische streken.

Twee casestudies: de Kempen en noordelijk Ghana.

OUDJAROU Lahcen: Lutte contre l'habitat sous-intégré au Maroc. Cas des centres du NordEst de Casablanca.

31 VANDE WIELE Tom: De invloed van waarnemingspatronen op de prestaties van het dubbele-Fourier-reeksen-model, het inverse-distance-model en het kriging-model bij het karteren van zandbanken.

\section{3}

VRYDAGHS Luc: Studies in opal phytoliths. Methods and identification criteria. 

Wadi Mujib basin (Jordan). A regional study based on field research, supported by map analysis and remote sensing, and integrated in a GIS.

\section{- Université Catholique de Louvain} Approach to Identify and Quantify Causes of Fire.

\section{- Université de Liège}


HILLALI Mimoun: La politique touristique marocaine.

OZER Pierre: Les lithométéores en région sahélienne: un indicateur climatique de la désertification.

WASTIAUX Cécile: Facteurs hydrologiques de la dégradation des tourbières hautes à sphaignes des Hautes Fagnes (Belgique).

WILLEMS Luc: Phénomènes karstiques en roches silicatées non carbonatées. Cas des grès, des micaschistes, des gneiss et des granites en Afrique sahélienne et équatoriale.

\section{1}

KABAMBA Kabata: Relations à la ville et territorialité dans la campagne environnante de Kananga (R.D. Congo).

\section{2}

BILLEN Roland: Nouvelle perception de la spatialité des objets et de leurs relations. Développement d'une modélisation tridimensionnelle de l'information spatiale.

BOUMEAZA Taieb: Le cours inférieur de la Moulouya. Morphologie quaternaire, dynamique fluviale et morphologie côtière du littoral entre Kebdana et Saidia (Maroc oriental).

OULHAJ El Bachir: Étude et modélisation de l'influence du réseau routier régional sur les eaux de ruissellement dans un bassin hydrographique. Applications aux bassins de l'Amblève, de la Vire et du Ton.

\section{3}

NDAYIENGURUKIYE Godefroid: Belief-based fusion framework for land cover discrimination: a synergistic approach with SAR and VIR satellite images.

NOTI N'SELE-ZOZE Joseph: L'impact de la fonction administrative sur le développement de la Ville de Bandundu (RDC).

TSHIUNZA Kalala: Mutations d'un système spatial rural. Cas du territoire luba kasayi.

\section{- Université Libre de Bruxelles}

\section{9}

CORNUT PIERRE: La circulation de l'eau potable en Belgique et à Bruxelles. Enjeux sociaux de la gestion d'une ressource naturelle.

\section{0}

CHAREF Mohammed: La circulation migratoire marocaine.

KHAZENDAR Ala: Marine ice formation in rifts of Antarctic ice shelves. A combined laboratory study and modeling approach.

\section{1}

VAN CRIEKINGEN M.: La rénovation résidentielle à Montréal et à Bruxelles. Dynamiques, impacts sociaux et rôle des pouvoirs publics.

\section{2}

ABOU-El-FARAH Y.: Tiznit et sa région.

MAZZINE H.: L'artisanat marocain du traditionnel au moderne. Le cas de Marrakech, Fès, Rabat et Salé. 
2003

KERBOUT Mohamed: Communautés rurales et gestion des ressources agro-pastorales dans le Moyen Atlas septentrional et ses bordures (Maroc).

AIT HASSOU Mohamed: L'intervention de l'état dans le secteur du logement au Maroc, et notamment à Marrakech. Le cas du Hay Al-Massira.

\section{- Vrije Universiteit Brussel}

\section{8}

PATTYN Frank: Ice sheet dynamics in eastern Dronning Maud Land, Antarctica. 1999

CANTERS Frank: Semi-automated map projection design. New ways to facilitate and improve small-scale map projection selection.

2000

KERZAZI Moussa: La migration rurale et ses incidences socio-économiques au Maroc

(Cas du Maroc oriental, du Gharb et de Témara).

2002

DIMANE Fouad: Contribution méthodologique à l'aménagement et la gestion des bassins versants au Maroc. Cas du bassin versant de l'oued Laou-Rif Occidental.

NYAKWEBA Enosch Osoro Elijah: Sustainable Tourism Development at the Kenya Coast.

2003

ABDUL LATIF Abdul Sahib Hussain: Quaternary (and partial pre-quaternary) aspects of the gology of the Bekaa valley -Lebanon.

\section{Mémoires de licence - Licentieverhandelingen}

\section{- Katholieke Universiteit Leuven}

\section{9}

BASTIAENS Jeroen: Stedelijke transformatieprocessen en post-apartheid, getoetst aan de structuur van Stellenbosch (Zuid-Afrika).

BRUYNINCKX Benjamin: Industriële ontwikkeling van Vlaamse en Noord-Franse passagiershavens, bij uiteenlopende institutionele kaders en beleidsopties. Case-study: een vergelijking Oostende-Calais.

BRYON Jeroen: Waterfrontontwikkeling in de vissershaven van Zeebrugge.

CASSIERS Tim: Etnisch ondernemen in een transnationale ruimte. De Brabantstraat in Brussel.

CLAES Kim: De evolutie van het bodemgebruik in post-apartheid Zuid-Afrika; feiten en percepties. Case-study: het Kleinhoevegebied ten zuiden en oosten van Bloemfontein.

DAENEN Vicky: Sociaal-economische en morfologische veranderingen in de Vlaamse begijnhoven vanaf het einde van de Franse Revolutie? 
DECRUYNAERE Leen: Nauwkeurigheidsanalyse van historische kaarten. Toepassing op de «Vlaanderenkaart» van Mercator (1540) en Ortelius (1750).

DE MAESSCHALCK Filip: Electoriale geografie van het Vlaams Blok. De ruimtelijke evolutie van de verkiezingsuitslagen van 1981 tot en met 1995.

DE TURCK Annemie: De invloed van de verschillende asielwetten op de spreiding van de kandidaat-vluchtelingen in België.

De VADDER Els: «Antwerpen-Zuid»: de veranderingen van een stadswijk van 1970 tot nu.

DE WEERDT Birgit: Achteruitschrijdingssnelheden van ravijnhoofden in Zuidoost-Spanje: een dendochronologisch onderzoek.

GILLIJNS Katleen: Kenmerken en Genese van gesloten depressies in Midden-België.

HULENS Ilse: Oostende: evolutie van badstad tot cultuurstad.

JACOBS Ellen: Samenhang tussen, en persistentie van klimaatkenmerken te Ukkel en de luchtbeweging boven Europa in 1881-1998.

JANSSENS Annelies: Aanbodstructuur van de detailhandel en consumentengedragingen in het stadsgwest Antwerpen.

LEMMENS Tim: Vergelijking en toepassing van verschillende datafusietechnieken voor teledetectie.

LOOPMANS Maarten: Ruimtegebruik en structuur van sociale netwerken: een case-study bij drie bevolkingsgroepen in de wijk Oud-Berchem (Antwerpen).

4 MAES Stéphanie: Cultuurtoerisme en revitalisatie in achtergestelde buurten. Case-study: Gent.

NAEDENOEN Ellen: Impact van economische evolutie in een traditionele industriële regio op woon- en bewonersstructuur. Case-study: de Rupelstreek.

ROMBAUT An: Het gebruik van fosfaten als tracer voor bodemerosie en -sedimentatie.

ROUFFA Katleen: Toerismeplanning en -beleid van archeologische sites in Turkije. Casestudy: Assos.

SERRUYS Philippe: Ruimtelijke spreiding van grondverschuivingen in de Vlaamse Ardennen.

SIX Simon: Bewerkingserosie in Zuidoost-Spanje: resultaten van een ploegexperiment op lange termijn.

TEMMERMAN Stijn: Merfodynamiek van zoetwaterschorren te Hingerene-Steendorp en getjieevolutie langs het Schelde estuarium.

TIERENS Nele: Bermravijnen langs de Rambla Torrealvilla (Zuidoost-Spanje): ruimtelijke spreiding en activiteit.

VAN BERENDONCKX Anke: Wisselwerking tussen terrein en luchtgesteldheid, toegepast op parapente.

VANDEKERCKHOVE Brecht: De Thurn en Taxisterreinen in Brussel: sterktes en zwaktes voor (her)ontwikkeling.

VANDE WALLE Stefaan: Toegankelijkheid tot sport tijdens en na de apartheid. Case-study: City of Tijgerberg. Cape Metropolitan Area, Zuid-Afrika.

VANMECHELEN Evi: Bejaardenmigraties in Zuid-Limburg. 
VANPAEMEL Linda: Massatransport in de Vlaamse Ardennen: ruimtelijke en temporele dynamiek.

VORMEZEELE Julie: Reliëfconstructies op archeologische sites; een case-study te TienenGrijpen.

WAUMANS Tine: Een regionale bodemerosierisicokaart voor Vlaanderen.

WIJNANT Joke: Residentiële mobiliteit in het Brussels stadsgewest. Een onderzoek naar determinanten en motivaties.

\section{0}

BOURGOIS Marie: Van kwaad naar erger? Waalse weekendverblijfplaatsen en wooncarrières van vaste bewoners in sociaal-ruimtelijke perspectief.

COLARDIJN Lieven: De Vlaamse technologievalleien: werkelijkheid of mythe?

DEMEULENAERE Sofie: Werkwijze voor het onderzoek naar de samenhang tussen blikseminslag en terreineigenschappen (met voorbeeld).

DENIS Liesbeth: Sociaal-ruimtelijke processen in Doel.

DE POOTER Bram: Milieuhygiënische locatieproblemen van huisvuilverbrandingsinstallaties in Vlaanderen: naar een samenspel tussen ruimtelijke ordening en milieubeleid? Case-study: Isvag-oven in Wilrijk.

DERVEAUX Heleen: De nationale en internationale betekenis van de haven van Antwerpen, mainport in de Hamburg-Le Havre range.

DEWILDE Anke: Migratiepatronen van asielzoekers in Europa met bijzondere aandacht voor Zwart-Afrikanen in België.

JANSEN Jeroen: Geowaarden: theoretische onderbouwing en praktische toepassingen in Limburg.

KONINCKX Xenia: Studie van de ruimtelijke variatie van sediment export in Spanje op basis van stuwmeeropvullingen.

LAVRYSEN Els: Studie van de ruimtelijke spreiding van stenigheid in een tropisch bergrivierbekken, Tigray, Ethiopië.

LUYTEN Edith: Ravijnvorming als gevolg van wegaanleg: een gevoelstudie in het hoogland van Ethiopië.

NEYENS Bart: De regionale ontwikkeling van Noord-Limburg: case study Bree.

NOORDMAN Annemie: De economische en ruimtelijke impact van een regionale luchthaven: case study Antwerp International Airport.

OP DE BEECK Nele: Een analyse van de overstroming van 1998 in het Netebekken tussen Lier en Heist-op-den-Berg.

SCHAERLAEKENS Wim: Agrarisch natuur- en landschapsbeheer als verbredingsstrategie.

VAN ELSEN Katrien: Integratie, emanicipatie en sociaal-ruimtelijke mobiliteit van allochtonen in huisvesting en onderwijs: de Turkse gemeenschap in de gemeente Mol.

VAN THUYNE Thijs: Laat-Holocene accumulatiegeschiedenis van het Bekken van Gravgaz (Sagalassos Territorium, S.-W.-Turkije).

WUYTS Ann: Binnenvaart als oplossing voor de problematiek van het goederenwegransport in België? Case study: het Zeekanaal Brussel-Schelde. 
BOULANGER Ankatrien: De biologische landbouw in België.

BROUWERS Els: De eigentijdse betekenis van sociaal toerisme. Case study: Limburg.

DEJONGHE Griet: Regionale ontwikkeling in het Meetjesland onder het Europees regionaal beleid en het vernieuwd Vlaams streekbeleid.

DE LAET Véronique: De opbouw en evolutie van de Aglasum-vallei, SW-Turkije.

D'HULST Gregory: Grondverschuivingen in de Vlaamse Ardennen: inventarisatie, mogelijke oorzaken en relatie met aardbevingen?

DONDERS Elisa: Een sociale geografie van de Brusselse volkstuinen.

HEUTS Wouter: De alledaagse geografie van straatbewoners in Brussel: het gebruik van het formele en informele voorzieningencircuit volgens een tijd-ruimte analyse.

HOSTENS Dirk: Werkwijzen voor het bepalen van het aantal frontpassages.

LAMBRECHTS Jona: On- en offsite-effecten van bodemerosie door water: een fysisch en sociaal-economische benadering. Studiegebied: de Molenbeekvallei in Gingelom.

LATHUY Cédric: Ruimtelijke geautocorreleerde fouten in digitale terreinmodellen: invloeden op zichtvelden.

LUYTEN Sarah: De invloed van de regionale economie op het rolpatroon. Een vergelijking tussen de Gentse textielregio en de Limburgse mijnregio.

QUAX Bart: Ruimtelijke variabiliteit van de overstromingssedimenten in de Suoi-Mai vallei, noordwest Vietnam.

SCHAERLAEKENS Leen: Sub-Sahara Afrika en Zuid-Afrika in de internationale economie en de voortrekkersrol van Zuid-Afrika.

SERNEELS Ellen: Evaluatie van het nut van ruilverkavelingen.

SIERENS Eveline: Herbestemming van het ruraal bouwkundig erfgoed in Limburgs Haspengouw - Toerisme: een oplossing voor het vrijkomend patrimonium?

STUYCK Karen: De invloed van de regionale economie op het rolpatroon. Een vergelijking tussen de Gentse textielregio en de Limburgse mijnregio.

VAN AUTGAARDEN Els: Implementatie van bodemconservatietechnieken in de Belgische Leemstreek gezien door de ogen van de landbouwers.

VAN DEN BOSSCHE Nathalie: Onderzoek van duinzanden uit Egypte.

VANDENREYKEN Helga: Ruimtelijke en temporale variaties van neerslag en zijn erosiviteit in een tropisch berggebied, noord-Etiopië.

VANDERSCHAEGHE Michiel: Typering en modellering van hellingsbewegingen in een klein bekken van de oge Ecuadoraanse Andes (Sta-Anna, Cuenca, Ecuador).

VERCAMST Eva: Herbestellingsmogelijkheden van 19de eeuwse industriële panden binnen het kernstedelijk weefsel. Case study: Gent.

VERHULST Klaas: Winkelgebieden en hun onderlinge concurrentie, toegepast op het stadsgewest Antwerpen o.b.m. het multi-attribuut attitude model.

VEYRET-PICOT Maude: Ravijnontwikkeling en -erosiecontrolemaatregelen in een tropisch bergrivierbekken: Tigray-Ethiopië.

VOS Gwendy: Waarderingsmethoden bij geowaarden - met toepassing op Zuid-Limburg.

WILLEMSENS Eef: De betekenis van de belforten voor cultuurtoerisme in Vlaamse steden. 
2002

ALDERWEIRELDT Karen: Residentiële spreiding en integratie van Zweden en Finnen in het Brusselse stadsgewest.

BIESMANS Marijke: Bodemverlies door het rooien van suikerbieten en aardappelen: ruimtelijke variatie op perceels- en regionaal niveau.

BLOEMEN Sylvie: De recreatieve ontsluiting van natuurreservaten. Studiegebied: Limburg.

COOREVITS Lieve: Tijd ruimtegeografie van alleenstaande moeders in de rand van Gent.

DEDOBBELEER Leen: A study of land use and its controlling factors of the Suoi Muoi catchment, in the mountain karst of North West Vietnam. Based on arial photographs.

DESCHEEMAEKER An: De evolutie van de sociale huursector in het Brussel Hoofdstedelijke Gewest.

GYSEN Annemie: Speelruimte voor kinderen in Antwerpen - relatie met stadsvlucht van jonge gezinnen.

LENAERTS Maarten: De geografische spreiding van de naturalisatie in België: Italianen, Spanjaarden, Turken en Marokkanen.

MOORS Ruth: Een vergelijking tussen verschillende exemplaren van de Kabinetskaart van de Oostenrijkse Nederlanden.

PIETERS Marieke: Woonmilieu en socialisatie van jongeren. Een vergelijking tussen de suburbane en de migrantenbuurten van het Brusselse stadsgewest.

SLEGERS Katrien: Een tijd-ruimtegeografie van asielzoekers: een case-study in het Brussels stadsgwest.

TIELEN Sofie: Duurzame ontwikkeling van toerisme in nationale parken en natuurgebieden in Zuid-Afrika.Het Golden Gate Hoogland Nationaal Park.

TIESTERS An: Analyse van de socio-economische, biofysische en bodemkundige factoren die de evolutie in de gedegradeerde gebieden van de zuidelijke Ecuadoriaanse Andes controleren. Gevalstudie: het stroombekken van de Machangara-rivier.

VALLONS Annemie: Onderzoek naar de historische en huidige typologie van Vlaamse rivieren in het kader van een hermeanderingsproject: casestudy van de Kleine en de Grote Nete.

VAN DEN EECKHAUT Miet: Kenmerken van en ontstaansvoorwaarden voor ravijnen onder bos: een gevalstudie in het Meerdaalwoud.

VAN ESCH Leen: Ruimtelijke analyse van de invloed van bodemgebruiksveranderingen op de riviermorfologie van en de sedimentdynamiek in eengebergterivier. Gevalstudie: het stroomgebied van de Deleg rivier, Hoge Ecuadoriaanse Andes.

VER GUCHT Joeri: Ruimtelijke impact van het toerisme in de binnenstad van Brugge.

VREVEN Bénédicte: De wooncarrière van Turken in Belgische stadsgewesten.

\section{3}

BAUWENS Lien: Intermodaal transport en de locatie van spoorgerichte intermodale terminals in België: een evaluatie o.b.v. netwerkanalyse.

BOUVEROUX Elke: De fruitstreek Zuid-Limburg-Hageland, een geografisch agribusinesscomplex? 
BRIJS Liesbet: Naoorlogse mobiliteitsontwikkelingen in relatie tot het wonen: gevalstudie: Tielt-Winge.

COEN Joke: Water- en modderoverlast in Sint-Truiden en Guigelom: een temporele analyse.

DE BAETS Sarah: De landschapsstudie op het overgangsgebied dekzand-polder (Moerbeke-Stekene-Axel-Hulst).

DEBBAUT Lies: De invloed van de buurt op de maatschappelijke integratie van Turkse vrouwen. Vergelijkende studie tussen Oud-Berchem (Antwerpen) en de Baarsjes (Amsterdam).

DE BRUYN Tom: Opslibbing van recent gevormde schorren in het Schelde-estuarium: een terreinstudie en modelevaluatie.

DEMUZERE Matthias: Thermodynamisch model van 2 Oost-Antarctische kustgletsjers.

DE WIT Joke: Stuwmeren in Tigray (Noord-Ethiopië): kenmerken, sedimentatie en sedimentbronnen.

KNAEPS Els: De mens in de laatste Ijstijd: dateringen als bewijsmaterial voor de bevolkingsbewegingen in West-Europa.

KNAPEN Anke: Ruimtelijke en temporele analyse van grondverschuivingen in Manjiya county, Mount Elgon regio, Oost-Oeganda.

KNOCKAERT Alysse: Duurzame effecten van evenementen: case studies Expo '92-Sevilla en Expo '98-Lisboa.

LAUWAET Dirk, Ruimtelijke analyse van de sedimentatie op een zout schor.

LENTACKER Kris: Kwantitatieve analyse van het reliëf in de vallei van de Aglasun Cayi, Turkije.

LEYS Roosmarijn: Hoevetoerisme als overlevingsstrategie voor de Belgische landbouwer.

MAES Herlinda: Pleinen en parken in Mechelen.

MULDERS Stijn: De landschapsvormende factoren in de gemeente Laakdal.

PASSCHIJN Maaike: Analyse van de geografische lokalisatie van de Midden-Neolitische boerenbevolking in België.

RIBUS Veerle: Reikwijdte van nieuwe leisure shoppingcentra - case study: Maasmechelen.

SANNEN Marijke: De betekenis van het zoekentoerisme voor de hotelsector in de Antwerpse Kempen.

SCHROOTEN Anne: De invloed van de bodemerosie op het bodemgebruik in het Hageland.

VANACKER Pascaline: «New Regionalism» en de invloed van EU-subsidiëring op de (her)ontwikkeling van de West-Midlands met focus op Birmingham.

VAN DESSEL Wim: Toepassingsmogelijkheden van ravijnrosiebestrijding: efficiëntie en effectiviteit van dubbele inzaai van wintergranen in de Belgische Leemstreek.

VANDROMME Lindy: Impact van bewortelingskenmerken op erosieweerstand van meige bodemtoplagen.

VANLOO Ans: Analyse van het Hageland als toeristische regio.

VITSE Ilse: Studie van gesloten depressies in Meerdaalwoud en omgeving.

VLIEGEN Senne: Sociale effecten van migraties in Indonesië, met case-study in West- en Centraal Kalimantan. 


\section{9} rondom Geel. Sai (Soedan). (Griekenland).

VRAL Mattias: Stedelijke ontwikkeling en elektorale evolutie: op zoek naar verbanden. Case study: Antwerpen.

WAUTERS Joop: Het ruimtegebruik van foorkramers in Vlaanderen: een sociaalgeografische interpretatie.

\section{- Rijksuniversiteit Gent}

\section{OPTIE GEOGRAFIE}

BRUYLANDT Ilse: Studie van het fysisch milieu van Santorini. Bijdrage tot de kennis van de regionale geografie van de Cycladen (Griekenland).

COEIMANS Steve: Economisch-geografische analyse van de industriële activiteiten in en

DANGREAU Geert: De fysisch-geografische kenmerken van de Belgische kust in het kader van de kustverdediging en het beleid.

DE COLVENAER Isabelle: Geografie van de verkeersongevallen in België.

DE HERDT Vincent: De stedelijke problematiek: een geografische analyse van de gemeentelijke begrotingen in het stadsgewest Gent.

DE KETELAERE Marina: Geomorfologische kenmerken van grotten in België.

DE MAN Joris: Onderzoek naar de mogelijkheden van Corona-satelliet-data als alternatief voor conventionele luchtfoto's in het kader van geo-archeologische studies, toegepast op

DE MEULENAER Klaartje: Studie van de menselijke invloeden op het fysisch milieu van Santorini. Een bijdrage tot de kennis van de regionale geografie van de Cycladen

DE RAES Wout: Studie van de geografische mobiliteit van de in het Brugse stedelijk leefcomplex gevestigde bedrijven, met de nadruk op de op bedrijventerreinen gelokaliseerde secundaire en tertiaire activiteiten.

DE REU Kirsten: Geografische aspecten van de uitzendsector in België, met een specifieke toepassing op Gent.

DESAEVER Jo: Geografie van het Wereldtoerisme.

DE SCHRIJVER Hans: Geomorfologische studie van de randzone van het Kymi-bekken. Een bijdrage tot de kennis van de regionale geografie van Euboia (Griekenland).

DURNEZ Dimitri: Studie van de kustmorfologie van het schiereiland Paximadhi. Een bijdrage tot de kennis van de regionale geografie van Euboia (Griekenland).

GARRE Nathalie: El Niño-Southern Oscillation. Bijdrage tot de kennis van de algemene structuur en de directe en indirecte gevolgen.

KLINCK Bart: De samenstelling van het bos in het laat-Eemiaam. Paleocologisch onderzoek op basis van houtanalyse van de afzettingen van Beernem.

KUPPENS Lies: Studie van «Fysisch milieu en Ontwikkeling» van de Meteora. Een bijdrage tot de kennis van de regionale geografie van Thessalië (Griekenland).

OST Lieven: Geomorfologische studie van de zuidoostelijke flank van het Ochi-massief. Een bijdrage tot de kennis van de regionale geografie van Euboia (Griekenland). 
PERSOONS Valentine: Kenmerken en dynamiek van het landschap van het Pays de Licques (Boulonnais, Pas-de-Calais, Frankrijk).

VAN BROECKHOVEN Barbara: De invloed van de omgeving op de esthetische belevingswaarde van het monument. Gevalstudie: Molens in het landelijk gebied.

VAN COILLIE Jelle: Geomorfologische studie van de Noordoostelijke flank van het Ochimassief. Een bijdrage tot de kennis van de regionale geografie van Euboia (Griekenland).

VAN DER STOCK Leen: De spreiding van het voltijds gewoon secundair onderwijs in Vlaanderen sinds het schoolpact.

VANDERSTRAETE Tony: Studie van «Fysisch milieu en Ontwikkeling» in de Mekong-delta. Een bijdrage tot de kennis van de regionale geografie van Vietman.

VAN DE WEGHE Nico: Geografische Analyse van de «Reisbureausector» in België met toepassing op het Gentse Stadsgewest.

VAN DE WIELE Hannelore: Evolutie en spreiding van de gesloten waters in het landschap aan de hand van kaarten en luchtfoto's. Een gevalstudie in de omgeving van Zomergem.

VAN HOLM Karen: Studie van het paleohydrografisch netwerk in de Tassili N'Ajjer (Algerije) aan de hand van Landsat MSS beelden en GIS.

VAN NIEUWENHOVE Koen: Geomorfologische studie van Geraardsbergen en omstreken.

VANRAEFELGHEM Eddy: Economisch-geografische aspecten van het Toerisme aan de Vlaamse Kust.

VERHOEVE An: Een geo-archeologische studie van het schiereiland Paximadhi. Een bijdrage tot de kennis van de regionale geografie van Euboia (Griekenland).

VONCK Yvan: Geomorfologisch en sedimentologisch onderzoek in het kader van de geomorfologische kartering van het kaartblad Kieldrecht-Lillo (7/5-6).

WILLEMS Isabelle: Studie van de ontwikkeling van het landgebruik in het Kagerabekken van Rwanda in relatie tot de eutrofiëring van het Victoriameer. Een bijdrage tot de kennis van «Fysisch milieu en Ontwikkeling», in het Nijlbekken.

OPTIE LANDMEETKUNDE

COEN Liesbeth: Transformatie tussen WGS84 en ED50.

DE TROYE Annelies: Installatie en inpassing van een permanent bifrequent Real-time GPS referentie-station in het EUREF netwerk.

HUTSEBAUT Koen: Het gebruik van Kriging bij kartering van zandbanken.

LESAGE Michaëlo: Verwerking van GPS-meetgegevens met Bernese Software.

NECKEBROECK Sofie: Vergelijking van topografische CAD-softwarepakketten: AutoCad, CivilCad, LisCAD en Pythagoras.

PERDAEN Steve: De Alsembergse steenweg bekeken vanuit een geografische invals-hoek met de nadruk op Ukkel.

VAN DAMME Frederik: Naar een reconstructieve typologie van de bebouwde ruimte in de provincie Oost-Vlaanderen.

VAN DEN BERGH Katrien: Ruimtelijke planning door het Vlaamse en het Waalse Gewest: een vergelijkende studie.

VAN DE SOMPELE Imme: De ruimtelijke impact van de tweede spoorontsluiting van de haven van Antwerpen. 
2000 Geel. (Griekenland). België.

VAN MAELSAEKE Stijn: Software-ontwikkeling voor Real-time Metingen van hoge nauwkeurigheid in de industriële metrologie, inclusief kwaliteits-controle.

VANRUTTEN Bart: Analyse van implementaties van algoritmen voor generatie van perspectiefzichten van vectoriële digitale terreinmodellen.

OPTIE GEOGRAFIE

BALCAEN Leen: Onderzoek van de vegetatieveranderingen over een periode van 1984 tot 1999 aan de hand van NOAA-AVHRR en Landsat gegevens in Namibië.

BEYAERT Veerle: Bijdrage tot de studie van de fysische geografie van de omgeving van het Itasy-meer in Centraal-Madagascar.

COEIMANS Steve: Een economisch-geografische analyse van de industriële activiteiten in

DALEWYN Sammy: Onderzoek van multi sensor en multi schaal beelden voor het herkennen van verschillende geomorfologische vormen, toegepast op het eiland Sai en omgeving-Soedan.

DEBAERE Renzo: Een socio-economische studie van Avelgem.

DE COLVENAER Isabelle: Geografie van de verkeersongevallen: een probleemstelling.

DE HERDT Vincent: De stedelijke problematiek: een geografische analyse van de gemeentebegrotingen in het stadsgewest Gent.

DE KETELAERE Marina: Geomorfologische kenmerken van grotten in België.

DE MEULENAER Klaartje: Studie van de menselijke invloeden op het fysisch milieu van Santorini. Bijdrage tot de kennis van de regionale geografie van de Cycladen

DEPREZ Cindy: Kartering en studie van de evolutie van de granulometrische parameters van bepaalde testgebieden op de Gootebank en de Kwintebank.

DERUDDER Pieter: Bijdrage tot de studie van de geomorfologie van Zuid-Jordanië.

DEVENTER Wouter: Geomorfogenetische studie van het Perlinkbeekbekken met implementatie van ecosysteemvisies.

DE VUYST Begga: Het stedelijk beleid ter bestrijding van kansarmoede in Gent toegepast op de Brugse Poort.

D'HAESELEER Erika: De geografische impact van de over-stromingen van september 1998 in het Scheldebekken.

GHYSELS Gunther: Studie van polygonale fossiele structuren in Vlaanderen op basis van luchtfoto's en veldonderzoek.

KUPPENS Lies: De impact van natuursport op het landschap met een gevalstudie in Hoog-

LOOMANS Tine: Het stedelijk beleid ter bestrijding van kansarmoede in Gent Gevalstudie: Sluizeken - Tolhuis - Ham.

MERTENS Karin: Geomorfologie en biodiversiteit van glaciale meren in de Alpen, toegespitst op de tijdelijke plassen van Gran Paradiso (Italië). 
RAVELINGIEN Bert: Laat-Kwartaire morfogenese en paleo-ecologie van een subantarctisch eiland. De gevalstudie van Vallée des Branloires (Ile de la Possession, Crozet, TAAF).

TINDEMANS Hans: Semi-perifere landen in het wereldsysteem: een poging tot nadere omschrijving.

VAN DE WIELE Hannelore: Evolutie en spreiding van de gesloten waters in het landschap aan de hand van kaarten en luchtfoto's. Een gevalstudie in de omgeving van Zomergem.

VAN DE WIELE Stijn: Bijdrage tot de kennis van de landschaps-geschiedenis van de Wase polders vóór de Farnèse-overstromingen.

VANMAELE Nele: Geomorfogenetische studie van het Poekebeekbekken met implementatie van ecosysteemvisies.

VAN NIEUWENHOVE Koen: Geomorfologische studie van Geraardsbergen (Kaartblad 30/6 en 30/7) met inbegrip van recente overstromingen.

VERDURMEN Els: Morfogenese en paleoecologie van de vallei van de beneden Kemmelbeek.

VERVAET Cedric: Bijdrage tot de kennis van het stedelijk klimaat met in het bijzonder aandacht voor de stedelijke warmte-eilanden problematiek.

WYDHOOGE Inge: Een bijdrage tot de studie van de fysische geografie en biogeografie van Oost-Turkije.

\section{OPTIE LANDMEETKUNDE}

BUERMAN Marieke (samen met Dirk VANDENBUSSCHE): Nauwkeurige real-time kinematische GPS-positionering voor hydrografische toepassingen geïntegreerd in 3Dseismisch onderzoek.

CALLAERT Dieter: Software-ontwikkeling (in een Visual C++, Windows CE omgeving) ten behoeve van een "palmsize» meetsysteem met real-time coördinaatbepaling via een mono GPS code-ontvanger gecorreleerd met geografische, geologische of topografische parameter-registratie.

COLLARD Christophe: Creatie van een hydrografische symbolenbibliotheek volgens de IHO specificaties op het cartografische systeem Mercator.

DEBUCQUOY Hans: Vergelijking van de overgangen tussen UTM en de Belgische Lambert.

DE CONINCK Frederik (samen met Frederic KNOCKAERT): Vergelijkende analyse van topografische vereffeningsoftware (Move 3, Star*Net) gesteund op eigen terreinmetingen uitgevoerd met waterpastoestel, totaalstation en GPS.

DE PESTEL Ludovic: De planbatenheffing in het decreet van 18 mei 1999 op de ruimtelijke ordening.

DE RUYVER Jo: Aspecten van de historiek en analyse van de methodiek van de oorspronkelijke kadasterkaarten met toepassing op enkele Oost-Vlaamse gemeenten.

DE RYCKE Sofie: Analyse van de bestaande ruimtelijke structuur van Eeklo.

DE WAELE Jan: Kaartproductie en -generalisatie: toepassing op het ontwerp van een kaartbeeld voor de numerieke reeks 1:100.000 uitgaande van het concept M737.

HENDRICKX Els: Vergelijkende studie van de ruimtelijke ordening rond Gent en rond Utrecht: gevalstudie Zuidwestelijke rand van Gent en Nieuwegein-Houten. 
LAPORTE Jan: Ruimtelijke Ordening en lawaaihinder rond luchthavens: Zaventem, Oostende en Deurne.

PAELINCK Mieke: Ruimtelijke analyse van het industriegebied «Stookte» te Wetteren, hierin begrepen de mobiliteitsproblematiek.

PERDAEN Steve: Verschuivingen in de bevolkingsamenstelling langsheen de Alsembergse Steenweg sinds 1981.

REUMERS Nele: 3D visualisatie in een stedelijk gebied aan de hand van digitale ruimtelijke gegevens. Bijdrage tot bruikbaarheid van digitale foto's bij de constructie van een 3Dmodel.

TACK Bram: De ruimtelijke analyse van het grensgebied Kortrijk-Lille.

VANDEN BUSSCHE Tomas: Ruimtelijke analyse van de infrastructurele corridor BruggeZeebrugge.

VAN LANCKER Hilde: Invloed van de maaiveldhoogte en de overstromingsduur op de vegetatie van het stedelijk natuurreservaat Bourgoyen-Ossemeersen. Onderzoek a.d.h. van gedetailleerde hoogtemetingen op een perceel met Kamgrasweide, Zilverschoongrasland en Scherpe-Zeggenvegetatie.

VANQUATHEM Tom: Corridors en het Ruimtelijk Structuurplan Vlaanderen.

VAN RYCKEGHEM Ann: Het Zwin: een fysisch geografische benadering.

VANSTEENVOORT Liesbeth: Satellietbeeldkaarten als basiskaart voor gebieden zonder recente cartografie. Gevalstudie. Realisatie van een kaart van Kivu (Congo) op 1/200.000.

WAUTERS Annemie: Vergelijkende analyse van topografische software pakketten, gesteund op eigen terreinmetingen - Pythagoras, Octopus-topo, Star-top.

\section{1}

OPTIE GEOGRAFIE

BISSCHOPS Kurt: Doel en Pernis: een vergelijkende studie.

BOSMANS Dirk: Geomorfologische studie van het Bekken van Kymi (Euboia, Griekenland). BOTERBERGH Bert: Onderzoek naar de integratie van CORONA KH-4A- en Landsat TMbeelden voor de detectie van bodemdrainageveranderingen in sebkhagebieden in de periode 1967-1994, toegepast op de provincie Ismailia (Egypte).

BRACKE Elke: De rol en het gebruik van het openbaar vervoer te Gent.

BUNTINX Stijn: Een historische relictenkaart van Oostakker: bijdrage tot de inventarisatie van het landschapshistorisch cultuurpatrimonium.

BURSENS Katrien: Inventarisatie van de oeverstructuren van de Gentse binnenwateren i.f.v. natuurlijk oeverbeheer.

CLAESSENS Ilse: Landschapsgenetisch onderzoek van de vallei van de Beneden-Schelde in de omgeving van Moerzeke.

DECLERCK Bert: Bijdrage tot de 3D visualisering: voorstelling van de toren van de kerk St. Victor in Fleurus.

DE MEYER Sarah: Detectie en kartering van de zeebodemmorfologie met behulp van ERSSAR beelden.

DE MULDER Griet: Het stedelijk beleid ter bestrijding van kansarmoede in Gent: een geval van governance? 
DEPREZ Marieke: Bijdrage tot de cartografie ten behoeve van de luchtvaart.

DUCHI David: De Noord-Atlantische Oscillatie: Concept en Impact op Europa.

GOETHALS Tanja: Een bijdrage tot de studie van de fysische geografie en geo-archeologie van het bekken van de Potenza-rivier (Prov. Di Macerata, Regione delle Marche, Italia).

HERSSENS Bram: Het electoraal succes van het Vlaams Blok op Gemeentelijk Niveau. Een electoraal geografische bijdrage.

HEYMANS Peter: De spoorweg in het landschap: een case-studie Dendermonde-Gent.

HOORELBEKE Mike: Democratie en vrijheid in de wereld: een geografisch overzicht.

HUYGHEBAERT Isabel: Windklimaat in België.

KREKELS Joke: Vergelijking van het grens-overschrijdend bocagelandschap van de Avesnois (Frankrijk) en de Fagne-Calestienne-Rièzes (België).

LAMBRECHT Barbara: Studie van de vegetatieveranderingen in oostelijk Kivu (D.R. Congo) aan de hand van Landsat TM- en ETM+-beelden.

LÖSKEN Kristoff: Onderzoek naar de mogelijkheden van radarbeelden (ERS-II) voor de detectie van bodemsaliniteit en waterverzadiging toegepast op de provincie Ismailia (Egypte).

MORTIER Frederic: Onderzoek naar de ruimtelijke ontwikkelingen in het gebied tussen de Leie en de E17 van Kortrijk tot Waregem.

PERSYN Jeroen: Diffussie van het Rode Kruis in Vlaanderen.

PODEVYN Els: Bijdrage tot de studie van de bevolking en de bewoningssites van het oostelijk deel van het eiland Naxos (Cycladen, Griekenland).

ROGIERS Katleen: Bosevolutie in Vlaanderen sedert Ferraris aan de hand van digitale kaartbestanden en GIS.

ROGGE Elke: Bijdrage tot de studie van het cultuurlandschap van het eiland Paros (Cycladen, Griekenland): nederzettingsanalyse en evolutie van de toeristische impact (1970-2000).

SEGERS Stijn: Multitemporele analyse van Corona-Landsat TM- en ETM+-beelden voor de detectie van bodemsaliniteit ten zuiden van Zagora (Vallei van de Draa, Marokko).

STEENHOUT Kristel: Een vergelijking van landschapsevaluaties voor het «Scheldeveld» ten zuiden van Gent.

THOEN Ad: Geomorfologische studie van het Massief van Ochtonia (Kymi, Euboia, Griekenland).

VAN DER HEYDEN Veerle: Ruimtelijke dynamiek van de tuinbouwbe-drijven in het Gentse toegespitst op Lochristi.

VANHAUWAERT Tine: Bijdrage tot het gebruik van kaarten en luchtfoto's voor de studie van de evolutie van de bebouwde oppervlakte.

VANHEVEL Ans: Bijdrage tot de studie van het cultuurlandschap en de bevolking van het westelijk deel van het eiland Naxos (Cycladen, Griekenland).

VANNEUVILLE Wouter: De studie van vage grenzen in het landschap d.m.v. GIS.

VAN ROSSUM Koen: Een historische relictenkaart van de gemeente Letterhoutem. Bijdrage tot de inventarisatie van het landschaps-historisch cultuurpatrimonium. 
VERFAILLIE Els: Bijdrage tot de studie van het fysisch milieu van het eiland Paros (Cycladen, Griekenland) aan de hand van terreinwerk, literatuur en GIS.

VIAENE Tom: Het «Deurganckdok» in paleogeomor-fologisch verband.

WARREYN Manu: Subatlantische fysische landschapsreconstructie in de omgeving van Oudenburg-Zandvoorde: probleemstelling bij de stratigrafische positie van archeologische vondsten.

WILDEMAUWE Tine: Multitemporele analyse van Corona-, landsat TM- en ETM+-beelden voor de detectie van eolisch transport in het gebied van Fezouata (Vallei van de Draa, Marokko).

WILLAERT Bart: Multitemporele analyse van de bodemgebruiksveranderingen aan de hand van kaartmateriaal en teledetectiegegevens in Zuidelijk Rwanda.

OPTIE LANDMEETKUNDE

AERNOUTS Annemie: Vergelijkende studie van de ruimtelijke ordening rond Gent en rond Utrecht: gevalstudie zuidwestelijke rand van Gent en Zeist.

BENOIT Frank (samen met Dennis DEVRIENDT): Onderzoek naar de mogelijkheden van digitale fotogrammetrische methoden voor terrestrische stereoscopische opnamen, toegepast op de Ruïnes van het transept en het koor van de kerk van de Aulneabdij (Thuin, Henegouwen).

BOGAERT Peter: Het raadplegen van een kaartdatabank met behulp van Mapserving.

COLE Lieselot: De problematiek van de ruimtelijke ontwikkelingen langsheen de N60.

CUVELIER Tim: Onderzoek naar de mogelijkheden van Corona KH-4A satellietbeelden voor de studie van koraalriffen in de Rode Zee (Hurghada-Egypte) met behulp van fotogrammetrische methoden.

DEBUCQUOY Hans: Vergelijking van de overgangen tussen UTM en de Belgische Lambert.

DE WINTER Berlinde: De Ruimtelijke dynamiek tussen Aalst en Brussel toegespitst op mobiliteit.

VANHOVE Tanja: De bestemming van de Rijtmeersen in het gewestplan Oudenaarde.

VANLATHEM Raf: Bijdrage tot vectorsymbolisatie ter vervanging van rastercartografie toegepast op de ASM kaart van Eurocontrol.

VAN MELE Jan Pieter: Bijdrage tot de studie van de toepassing van GIS in het facility management aan de universiteit d.m.v. mapserving.

VAN PRAET Piet: Cartografische inventaris van overstromingen op basis van KMI gegevens voor de periode 1981-2000.

VANVINCKENROYE Christof: Onderzoek van historisch topografisch instrumentarium.

VAN WAES Griet: De problematiek van de mobiliteit van en naar de kleine kernen in het Meetjesland, ten noorden van de E40.

VERHOFSTADT Jan: Onderzoek naar de fotogrammetrische parameters en restitutie mogelijkheden van Corona KH-4B data, toegepast op Wadi Mujib, Jordanië.

VERVAET Marc: Het gebruik van geografische informatie-systemen in havens. Een vergelijkende studie.

2002

OPTIE GEOGRAFIE 

Denemarken. ASTERbeelden. 1843-1914). op België.

BLINDEMAN Jacinte: Bijdrage tot de studie van het fysisch milieu van de «Regione delle Marche» (Italia) met behulp van satellietbeelden en terreinwaarnemingen in het kader van de «Potenza Valley Survey».

CALUS Karin: Reconstructie van Holocene ecosysteemveranderingen in het Tanganyikameer aan de hand van fossiele diatomeeën.

DEGEZELLE Leen: Landschapstypologie en de inventaris van autochtone bomen en struiken in het regionaal landschap West-Vlaamse heuvels.

DE GRAVE Veronique: De ontwikkeling en beleving van het industrieel landschap in noordoost Estland.

DE MUER Liesbeth: Migratie en asiel: hoe open zijn onze grenzen? Een ruimtelijke analyse van 10 jaar Belgisch asielbeleid.

DE PEUTER Bea: Biologische landbouw in het landschap, Vlaanderen vergeleken met

DEPREZ Sarah: Impact van de Hoge Aswandam op de benedenloop van de Wadi Allaqi

(ZO-Egypte): een multitemporele studie a.d.h.v. CORONA-, Landsat ETM+- en

DE WOLF Nele: Bijdrage tot de studie van de Italiaanse 16-de eeuwse samengestelde atlassen, i.h.b. IATO-408.

DE ZWAEF Jelle: De ontwikkeling van de eerste Belgische Basiskaart op 1: 20000 (pe-riode

D'HEER Laura: Bijdrage tot de studie van het «Fysisch Milieu en Ontwikkeling» van Beneden-Kongo (RDC).

HUYGHEBAERT Boris: Planning en Besluitvorming in Gent 1991-2001.

KERCKHOVE Steve: Studie van de windklimaten aan westeuropese kusten en toepassing

MEERSCHAUT Kristof: Ruimtelijke dynamiek van Ronse als grensstad in het spanningsveld tussen Vlaanderen en Wallonië.

MEYNEN Nick: Een methodologie voor het opvolgen van het landgebruik aan de hand van multitemporele luchtfoto's en satellietbeelden, toegepast op Ruanda en Oeganda.

MORTELMANS Jan: Ruimtelijke analyse en dynamiek van de Oostendse Oosteroever.

MOULAERT Ine: Geomorfologische studie van de kust van het Albères-Massief in Frankrijk (Pyrénées Orientales).

NOENS Pieter: De duinendecreten: een politiek-geografische analyse.

PELGRIMS Hilde: De overstroming van Ruisbroek 1976: een geografische en maatschappelijke benadering.

QUINTELIER An: Woonwensen van jongeren in Vlaan-deren met betrekking tot het zelfstandig wonen: een sociaal-geografische studie.

RAES Liselotte: Bijdrage tot de studie van de geomorfologie en geoarcheologie van het gebied rond Passo Di Treia (Provincia Di Macherata, Regione Delle Marche, Italia).

REYNS Johan: Kartering van de morfodynamiek met behulp van multibeamwaarnemingen voor de Schelde te Rupelmonde.

ROTTIERS Jeroen: Analyse van de parkeermogelijkheden in Gent. 
STERKEN Mieke: Veranderingen in het klimaat en landgebruik in de Cuzco-regio (Cordillera Oriëntal, Zuidoost Peru) tijdens het laatste millenium: een reconstructie d.m.v. diatomeeënanalyse.

VALCKE Steven: Regionale, sociaal-economische gevol-gen van de inplanting van een

belangrijk infrastructuurwerk; case study: Kanaaltunnel en West-Vlaanderen.

VAN DE MOORTELE Kevin: Kritische toetsing van het centrum-periferiemodel van Magee over voetbalmigraties.

VANDENDRIESSCHE Thomas: El Nino en de Zuidelijke Oscillatie: de relatie met de Noord Atlantische oscillatie, de Arctische oscillatie, de Antarctische oscillatie en de Noord Pacifische oscillatie.

VANDENHOUTE Lieselot: Studie van de vegetatiedynamiek ten zuiden van Kikwit (Bandundu-Democratische Republiek Congo) aan de hand van multitemporele satellietbeelden.

VAN DER BIEST Katrien: Bronnenstudie in Oost- en West-Vlaanderen vanuit geomorfologisch standpunt.

VAN DE VELDE Sara: Morfologische en functionele verstedelijking in de omgeving van Dendermonde (van Ferraris tot heden).

VANDROMME Jeroen: Verandering van het landschap van de Ardense Semois sedert het einde van de 18de eeuw.

VANOUTRIVE Thomas: Historiek van de cartografie van Katanga.

VAN HOOREBEKE Ken: Onderzoek naar de mogelijkheden van het gebruik van Zeer Hoge Resolutiedata (IKONOS) voor het detecteren van verkeersattributen.

VERMANDERE Irmine: Recente ontwikkelingen en perceptie van het landschap op het eiland Saaremaa (Estland).

2 VERMEIR Steven: De wet van Reilly en de spreiding van winkelketens in Vlaanderen en Brussel.

VERSLYPE Jeroen: Analyse en dynamiek van Middelkerke in de Oostendse Ruimte, een ruimtelijke positionering.

4 VERSTRAETE Lies: Culturele differentiatie en perceptie van het landschap van Setomaa in Zuidoost Estland.

OPTIE LANDMEETKUNDE

BESBRUGGE Steven: De kartering in België tijdens het Hollands Bewind (1815-1830): De hoofdtriangulatie van Kapitein Erzey.

DE BOCK Inti: Kwaliteitsbeoordeling van grondslagmetingen met totaalstation en differentiële GPS.

BE BOLLE Pieter (samen met Wim VAN BOGAERT): Analyse van de parameter die de kwaliteit bepalen van een gemengde kleinste kwadratenvereffening van topografische grondslagmetingen met GPS, totaalstation en waterpastoestel.

DE TEMMERMAN Leen: Historiek en vergelijkende studie van de Belgische schoolatlassen.

FAES Anne: Onderzoek van besteksconformiteit door controle op topografische detailmetingen met totaalstations en door analyse van het digitale grondplan (in Autocad). 
HEYMAN Jens: Zoektocht naar de grensoverschrijdende dynamiek van de as NinoveGeraardsbergen-Lessines.

MADDENS Ruben: Ontwerp en beheer van een webcarto-theek voor historisch kaartmateriaal: methodologie en toepassing.

MATTHYS Katrijn (samen met Liesje LOZIE): Onderzoek naar de positie van de camera bij digitale fotogrammetrische restitutie voor terrestrische stereoscopische opnamen en het opzetten van de productie van orthofotoplans toegepast op de kasteelruïne van Maria van Hongarije te Binche.

SCHEERS Stijn: Een ruimtelijke herprofilering van de mijnsites: een toepassing te Beringen.

VANBILLEMONT Bjorn: Implementeren van kaartdocumenten en luchtfoto's in een GISpakket toegankelijk voor archeologen.

VANDE KERKHOF Nico: Onderzoek naar de mogelijkheden van WESCAM-videobeelden, opgenomen vanuit helikopters, als alternatief voor conventionele luchtopnames.

VAN DEN BERGHE Erik: Onderzoek naar de invloeden van de ionosfeer en van multipath op GPS-metingen.

VAN HEUVERSWYN Jeroen: Toeristische en recreatieve schets van Gent in de Oostvlaamse en Vlaamse kontekst.

VAN QUAETHEM Veronique: De ruimtelijke dynamiek van het stedelijk gebied Tielt in zijn regio.

2003

\section{OPTIE GEOGRAFIE}

ADRIAENSSENS Karen: Bijdrage tot de studie van het fysich milieu van het eiland Milos (Cycladen, Griekenland).

BRAECKMAN Nathalie: De afvalproblematiek in Vlaanderen, toegespitst op bedrijfsafval (gevalstudie: Sidmar NV).

BUNNEGHEM Ann: De gezinsdynamiek binnen regionale woningmarkten. Drie gevalstudies: Gent, Brugge en Dendermonde.

BUYSSE Wim: Kinderen en jongeren in de stad. Case: ruimtelijke analyse van de speelruimte in Gent.

CLEENWERCK Dieter: Draagkrachtstudie van het Gents openbaar vervoersnet met behulp van netwerkanalyse.

DECKERS Pieter: Kartering van fysich geografische aspecten in een gebied rond de vierde cataract (Soedan) met behulp van ASTER en CORONA.

DE MEULENAER Lies: Onderzoek naar de lichthinder in het landschap aan de hand van een gevalstudie in het Land van Waas.

DENAERT Ellen: Lochristi en Wachtebeke in het spanningsveld van Gent: Stad en Haven.

DENOLF Charlotte: Een bijdrage tot de studie van de geomorfologie en de geo-archeologie van het mondingsgebied van de Potenza (Provincia di Macerata, Regione delle Marche, Italia).

DE PAEPE Bart: Contourieten langs het «Mediterranean Outflow Water». 
DE ROUCK Tinneke: De studie van toponiemen op tijdreeksen van kaarten en hun landschappelijke interpretatie. Een gevalstudie in het Pajottenland.

DERVEAUX Isabel: Woon-werkverplaatsingen in het arrondissement Dendermonde.

DE SADELEER Hadewych: Sociaal-economische kwaliteiten van Zottegem in de regio ZuidOost-Vlaanderen.

DE WACHTER Els: Verstedelijkingsprocessen in de periferie van het kapitalistische wereldsysteem.

DE WILDE Jeroen: Beslissingsondersteunende functie van een 3D visualisatie toegepast op de omgeving van het Rabot.

DORME Evelien: Impact van stuwdammen. Gevalsstudies in West-Afrika.

DUGERNIER Marten: Milos (Cycladen, Griekenland): van ruimtelijke analyse naar ruimtelijke planning.

FERRE Michaël: Ingreep van de urbanisering op de vallei van de Benedenschelde, stroomopwaarts Wetteren.

MARICOU Hans: Democratie in Afrika: een politiek-geografische studie.

NIEMANS Britt: Het verplaatsingsgedrag van de Gentse actieve bevolking geanalyseerd. Een activiteiten-patronenbenadering.

NOLF Geraldine: Bijdrage tot de studie van de evolutie van het cultuurlandschap van het eiland Milos (Cycladen, Griekenland): terreinwerk, bevolkingsanalyse, fotostudie en cartografische analyse (1972-2002).

NOTEBAERT Bastiaan: Gedetailleerd geomorfologisch karteren van vlakke gebieden: casestudie kaartblad Nevele.

SCHELFAUT Hilde: Vakantievoorkeuren gemodelleerd: «stated preference» benadering.

SOENEN Benoît: Studie over de ligging en evolutie van toponiemen op de kaart van Ferraris t.e.m. de derde basiskaart van het NGI in de regio Eeklo-Zomergem-Lovendegem.

TAVERNIERS Vincent: Geographical distribution of Mayawa vanilla farmers (Tanzania). Geografische spreiding van de Mayawa vanille boeren (Tanzania).

TEMMERMAN Stein: Mogelijkheden en problemen van postevaluatie bij MER voor monumenten en landschappen aan de hand van gevalstudies in de Gentse kanaalzone.

VAN ACKER Veronique: De profilering van Lochristi in de Gentse regio, aspect mobiliteit.

VAN COILLIE Stijn: Onderzoek naar de mogelijkheden van de Terra-satellietsensoren voor de aanmaak van relatieve en absolute DEM's voor de République Démocratique du Congo.

VAN DER AA Jörgen: De impact van de sluiting van de ring R42-Sint-Niklaas op mobiliteit en stedelijke dynamiek.

VANDERHEIDEN Stijn: Pijpleidingen vervoer: een geografische analyse.

VAN DER MEEREN Floor: Studie van de kustmorfologie van het Adriatische Zeegebied.

VAN DE WOESTYNE Veronique: Geografische aspecten van de prostitutie.

VAN DORPE Jolien: Zonevreemde bedrijven in Vlaanderen, casestudie ten noordwesten van Gent.

VERBEKEN Joris: Integratie van vroegere zendings-rapporten voor de kartering van de vegetatie in het Virunga-Park (D.R.C.) door middel van Landsat en Aster.

VERSTRAETE Eva: Ruimtelijke dynamiek van Brugge. Wonen versus toerisme. 

studie.

\section{9} Itradec. Sénégal. Burkina Faso.

WUYTS Vanessa: Kwantitatieve reconstructie van milieuveranderingen in Antarctische kustmeren (Lake Burgess en Lake Nella) tijdens het Laat Holoceen.

ZANDERS Joke: Vaubanvestingen langs de Frans-Belgische grens landschappelijk bekeken.

\section{OPTIE LANDMEETKUNDE}

DE MUNTER Griet: De problematiek van zonevreemd wonen. Theoretisch kader en beleidsaspecten met toepassing op Dendermonde.

DE ROUCK Kristien: Nauwkeurigheidsanalyse van historische kadasterkaarten, toegepast op enkele Oost-Vlaamse gemeenten.

DONOVAN Linton: Historiek van de ondergrondse topografische opmetingen met case

HENNAU Marc: Vergelijkende analyse van smoothing-algoritmes voor iso(hoogte)-lijnen en onderzoek van de efficiëntie van de geïmplementeerde algoritmes in Delphi.

MOORS Ruth: Een kwantitatieve en kwalitatieve analyse van de karteernauwkeurigheid op de grootschalige Ferrariskaart.

TAILLEU Kristof: Onderzoek naar de schaal bij fotogrammetrische restitutie bij de aanmaak van orthofotoplans, toegepast op de kerk «Sainte-Croix» te Luik.

VAN DEN BUVERIE Hans: Studie, ontwerp en implementatie van een topografisch kalibratielokaal.

\section{- Université Catholique de Louvain}

BOTTIN G.: Contribution à l'évaluation écologique du remembrement agricole en Région Wallonne; le cas du remembrement de Ville-en-Hesbaye (Province de Liège).

BRUTSAERT B.: L'influence des externalités négatives sur la détermination de la localisation optimale par le modèle de la p-médiane. Etude d'un cas: la localisation d'un centre de tri des déchets et d'une unité de biométhanisation au sein de l'Intercommunale

CARBONNELLE S.: Influence de la localisation géographique et de la plante hôte sur l'infestation par Tetranychus urticae Koch (Acari: Tetranychidae) en cultures de betteraves et de maïs.

DE HERTOGH A.: Etude par télédétection et enquête socio-économique de l'évolution du paysage agraire d'une communauté traditionnelle africaine. Cas de Médina Gounass,

DE KEYZER J.-L.: Essai d'analyse spatiale de la pauvreté à Bruxelles.

DE LE COURT A.: Etude de la structure spatiale de la criminalité. Essai d'explication de la répartition des cambriolages à Charleroi.

FANUEL H.: Le nouveau jeu de la concurrence air-fer en France.Application du modèle «distance-valeur du temps».

FOURNY B.: Cartographie de la végétation d'une tourbière: La Fange aux Mochettes (Samree/La Roche), du XVIIIe siècle à nos jours.

FRETIN J.: Etude par télédétection et SIG de l'impact écologique des migrants mossi au 


\section{0} géographique. agriculture. Ardenne belge. européenne. urbaine.

GILLET S.: Analyse de l'implantation d'un parc naturel: le parc naturel Viroin-Hermeton.

LELEUX L.: Analyse spatiale de la criminalité dans le Hainaut.

MEURICE M.: Analyse spatiale de la criminalité. Cas de six communes hennuyères.

NICOLAY B.: Les prix des maisons et des terrains à bâtir en région wallonne.

PARSY C.: La dégradation des forêts ouvertes en Tanzanie: le cas de Lugoba. Analyse par télédétection et par enquêtes socio-économiques

PEREMANS A.-C.: Discrimination spatiale des ménages monoparentaux dans l'agglomération bruxelloise face à la pauvreté en terme de logement.

VAN CALBERGH B.: Les impacts potentiels sur l'agriculture ardennaise d'une variation climatique due à l'intensification de l'effet de serre.

VAN DEN STEEN N.: Etude par télédétection de l'impact de la production de bois de chauffe sur le phénomène de déforestation et de dégradation des sols dans les pays en développement. Le cas de la communauté rurale de Médina Gounas, Sénégal.

BILLIET M.: Les flux téléphoniques interzonaux belges en 1998 : une approche

CAPIAU 0.: Un Système d'Information Géographique du réseau des chemins de fer belge.

COCLE D. : Implantation optimale d'un réseau d'assainissement d'eaux usées urbaines.

DAIX N.: Influence de la rériurbanisation sur l'offre de transports en commun.

DARON S.-P.: Impact of land use change on nitrous oxide emissions from European

DE VOGEL C.: Biogéographie et écologie comparée de deux espèces de sauterelles en

DERZELLE C.: La concentration régionale des firmes de haute technologie dans l'Union

DUJARDIN C.: Effet de frontière et interaction spatiale. Le cas des migrations alternantes et de la frontière linguistique en Belgique.

ECKHARDT N.: RER et demande de transport vers Louvain-la-Neuve.

EVERS J.: Exploration du canal 1,6 $\mu \mathrm{m}$ dans l'étude des écosystèmes tropicaux.

GENARD H.: Evaluation écologique et paysagère après remembrement agricole.Le cas de la commune d'Eprave en Famenne namuroise.

HANON O.: Contribution à l'étude comportementale de la couche limite atmosphérique

LEPERS E.: Analyse spatio-temporelle de la distribution du choléra au Mozambique entre 1981 et1995. Influence des conditions climatiques.

LUYCKX A.: Etude de la dégradation des forêts sèches en Tanzanie.Modélisation des activités de production de charb on de bois et évaluation des impacts.

MESTDAG C.: Impact de l'évolution de l'occupation du sol sur le débit provoqué par une pluie centennale pour le bassin versant du Ruisseau du Fond d'Arquet à Champion.

MEYFROIDT P.: L'impact des feux sur les changements de végétation: l'influence de l'utilisation du sol. 
PETRELLA-TIRONE E.: Les mutations spatiales des activités dans l'agglomération opérationnelle de Bruxelles.

SERVAIS M.: Comparaison des méthodes de détection des changements pour les images satellitaires ATSR-2. Etude de cas en Côte d'Ivoire entre 1997 et 1998.

TIMMERMANS A.: La pauvreté à Charleroi.Typologie des quartiers et politiques du logement.

VAN LOQUEREN T.: Le chauffage au charbon est-il un indicateur de pauvreté?

VANWAMBEKE S.: Les changements d'occupation du sol dans la vallée du Zambèze: Le cas de la zone de Lusitu - Analyse par télédétection et photographies aériennes.

2001

BLAISE V.: Comparaison des structures socio-spatiales de Paris et Bruxelles.

BREESCH E.: Etude de la localisation optimale du centre de distribution du Groupe Mestdagh situé à Gosselies.

DE HOUCK A.: Analyse et modélisation des changements d'affectation du sol dans le Brabant wallon.

DE LONGUEVILLE F.: L'influence de l'environnement naturel sur la décision de migrer à l'intérieur du Burkina Faso. Etude de cas sur base d'une enquête de ménages.

DEBUIGNE B.: Etude de l'intensification agricole au Malawi. Intégration de données multisources et politiques de reboisement.

DENIL B.: Analyse des formes spatiales de la zone périurbaine dans le cas de Namur et Bruxelles.

LEJEUNE S.: Impacts des changements d'occupation du sol sur la répartition d'espèces de faune sauvage au Masai Mara.

MALCHAIR A.: La concurrence air - fer en Allemagne.

PECHEUR C.: Analyse de l'aire de marché d'un centre de transbordement intermodal. Le cas de «Terminal Container Athus».

PIERSON M.: Proposition d'implantation d'un service de collecte et de traitement des déchets ménagers dans les provinces de Luxembourg et de Namur.

SCHMIT C.: Evaluation de techniques simples pour modéliser le ruissellement dans deux petits bassins versants en milieu semi-aride.

SCOUVART M.: Changements d'occupation du sol en Amazonie brésilienne: bases pour l'élaboration d'un modèle de simulation dynamique.

STEVENS A.: L'impact du labour intensif sur la distribution des sédiments en région semiaride: le cas des cultures d'amandiers en Espagne du Sud-Est.

VAN DEYNZE L.: L'accessibilité ferroviaire en Belgique: les planifications Ic/IR successives de la SNCB.

2 VANDEVOORDE J.: Recherche de la localisation optimale d'une gare de type «Parkway» pour matériels à grande vitesse, le long de la ligne nouvelle joignant Bruxelles à la frontière française.

VERMER F.: La rétroaction de la végétation sur le climat de l'Holocène; étude à l'aide d'un modèle climatique. 

au Honduras. management. économiques.

\section{9} Malchamps.

BARBETTE A.-C.: Modélisation par automates cellulaires d'une partie de la frange périurbaine de la ville de Bruxelles.

BAUGARD Y.: Contribution à l'étude comportementale de la couche limite atmosphérique et de la pollution en milieu urbain.

CORLAZZOLI M.: Changement de la couverture du sol après le passage de l'ouragan Mitch

DE TIEGE J.: Test du concept «Ecological Footprint» au Sahel et en Belgique.

DENDONCKER N.: Belgium's CO2 mitigation potential under improved agricultural

DUBUS B.: Inventaire des parcelles d'amandiers nivelées au bulldozer dans la région de Murcia, modélisation et conséquences de ce processus de nivellement.

FOHAL J.: L'impact de l'instauration du plan de secteur sur l'aménagement du territoire dans les communes de Rixensart et Lasne.

GHISLAIN C.: Etude de l'intensification agricole au Malawi par l'utilisation d'images satellitaires durant la période 1985-1995 et mise en relation avec des données socio-

LETERME B.: Distribution spatiale de la biomasse, des affleurements rocheux et des sols nus dans la Sierra de Gador: modèle de Spectral Linear Unmixing.

\section{- Université de Liège}

option géographie fondamentale

BONNI Didier: L'immigration allemande dans l'est de l'arrondissement de Verviers.

CHARLIER François: Typologie géomorphologique de linéaments reconnus par photointerprétation. Tentative de constitution et exemple d'exploitation d'un S.I.G.

DEWITTE Olivier: El Niño et l'oscillation australe. État de la question par analyse bibliographique.

DONNAY Yvan: Contribution à l'étude de la géomorphologie glaciaire dans la basse vallée de l'Arve (Haute Savoie, France).

ÉTIENNE Hugues: Contribution à l'étude des hydrométéores à partir des observations du temps présent. Comparaison des stations météorologiques d'Elsenborn et de Spa

FRAIPONT Sabine: La périurbanisation en Hesbaye. Les communes d'Incourt, de Verlaine et de Wasseige.

FRANÇOIS Sylvie: Contribution géographique à l'étude d'un problème sanitaire. Aperçu bibliographique de l'association entre la pollution atmosphérique, les paramètres climatiques et les maladies respiratoires chroniques des voies inférieures. Étude de cas dans la région liégeoise.

GOSSELIN Claire: Le creusement de la grotte «La Merveilleuse» de Dinant. Influence structurale et spéléogenèse.

HALLOT Éric: Karst dans les évaporites. L'exemple du Mont Sedom - Israël. 
LANNOY Catherine: Problématique de l'évolution de la localisation des immeubles de bureaux dans la ville de Liège depuis 20 ans.

LÉGAT Adrien: Apport de la télédétection dans l'étude de la désertification dans la vallée du Draa. La palmeraie de Fezouata.

LHOEST Geoffray: La localisation et l'évolution des clubs de football des divisions supérieures en Belgique pour les trente dernières années.

MAIRY Nicolas: Disparités régionales de la pénétration des télécommunications en Belgique. L'exemple des adresses électroniques.

SALMON Marc: Etude diachronique de l'évolution du littoral dans un milieu fortement urbanisé. Cas de la Baie de Mohammedia.

SCHÉNA David: Les disparités de consommation en eau de distribution. Étude appliquée aux régions wallonne et bruxelloise.

SMAL Christelle: Etude des forces et des faiblesses d'un territoire pour un développement local; un exemple: le plateau de Rocroi.

SPAILIER Fabien: Impact de la mutation de la localisation des complexes cinématographiques sur les comportements des clientèles. Le cas de Liège.

VALOIR Gilles: Etude du fonctionnement et de la localisation des fours à chaux artisanaux au Maroc et essai d'application au Fouta Djallon en Guinée.

WILMET Fabienne: Apports de la théorie de Dempster Schafer à la classification des images satellitaires. Application à la zone urbaine liégeoise.

option géométrologie

AUPAIX Michaël: onception d'un système d'information géographique pour le groupe T.E.C.

BROUWIER Michaël: La recherche d'une faille sur le plateau des Hautes Fagnes.

CLERFAYS Sandra: Détermination des indemnités relatives à la procédure d'extrême urgence en matière d'expropriation pour cause d'utilité publique.

DE CLERCQ Yves: Photogrammétrie satellitaire. Étude des concepts et évaluation des produits.

DUPUIS Emmanuel: Réception de données topographiques. Problèmes de référence, de complétude et d'exactitude de position planimétrique.

GOSSET Philippe: Étude des possibilités d'application de la technologie S.I.G. à la gestion et à l'entretien des voiries. Cas de la commune de Grâce Hollogne.

HICK Grégory: Topographie souterraine en $3 \mathrm{D}$. Application à la grotte de Remouchamps.

LAFAYE Serge: Levés topographiques en milieu souterrain. Application à la grotte de Comblain au Pont.

QUINTIN Jean François: Le marché des terrains à bâtir à Lincent et Hélécine.

SHEEREN David: La conception des bases de données spatio-temporelles. Problématiques et solutions dans le cadre du formalisme CONCOO.

\section{0}

option géographie fondamentale

DEHARD Rodolphe: Le karst de la région calcaire et dolomitique située entre Bomal et Hamoir. 
579

DERWAHL Gwendolin: Dépôts en grottes. Relations entre couleur et composition chimique.

GOB Frédéric: Application de la lichénométrie à l'étude d'un torrent corse, La Figarella. Étude de la dynamique sédimentaire récente du bassin et de la mobilisation de la charge de fond.

HOUBRECHTS Geoffrey: Utilisation des macroscories comme indicateur de transport de la charge de fond des rivières de la «Terre de Durbuy».

LANGE Stéphanie: Le contrat de rivière Ourthe: contribution méthodologique à l'élaboration de l'inventaire et à la délimitation des zones inondables.

MEUREAU Xavier: Le concept d'éco-quartier. Son application à la ville de Liège.

PETIT Sébastien: Analyse quantitative du réseau hydrographique de la Meuse: exploitation d'un modèle numérique de terrain.

PIRENNE Marie: L'agriculture biologique en Wallonie. État actuel et diffusion.

SILVESTRE Valérie: De la philosophie à la pratique d'un tourisme durable? Le cas des parcs naturels régionaux français. Essai d'application aux Cantons de l'Est.

option géomatique et géométrologie

BRIXKO Jérôme: Le code global, un système de référence universel (Applications à la Belgique).

HARDY Bruno: Étude du marché immobilier semi industriel.

JONET Lionel: Incidence morphodynamique de réaménagements de rivières: cas de l'Ourthe occidentale à Moircy.

KESSLER Laurent: Étude de coûts de viabilisation dans les lotissements publics en province de Liège.

LEGROS Gauthier: La cartographie sur Internet (Prototype d'application pour la recherche interactive d'itinéraire sur le campus universitaire du Sart Tilman).

PASQUASY Fabien: Standardisation des métadonnées géographiques (élaboration d'un outil de gestion pour les PPNC).

SAVOIE Grégory: Cartographie minière en Wallonie (Précision des plans de surface).

2001

option géographie fondamentale

BRICHET Hervé: Géographie des organisations sectaires internationales.

CHARLIER Anne: Apport des photographies aériennes de la Première Guerre mondiale à l'étude de la morphologie littorale des régions de Koksijde et de Ramskapelle.

COREXENOS Pavlos: Détermination des risques que présentent les terrils. Le cas du terril de Bernalmont.

GEERTS Véronique: La spatialisation de la criminalité. Une étude de cas: la criminalité à Liège de 1997 à 2000.

LEJEUNE Éric: Analyse des «flash floods» et des précipitations de forte intensité en Région wallonne.

2 MOLS Julien: Paramètres physico chimiques de sources et piézométrie dans le Crétacé du Pays de Herve et de la Hesbaye.

03 PEETERS Alexandre: Évolution du littoral du golfe de Sant'Amanza (Corse) depuis 1952. 
SCHYNS Jean Christophe: La palmeraie de M'Hamid dans la vallée du Drâa: étude des composantes de la désertification et apport de la télédétection à leur évaluation.

option géométrologie

BALANCIER Laurent: Étude des erreurs admissibles dans un levé de précision.

BAVIER Michaël: Influence du fenêtrage sur la précision des mesures G.P.S.

BEAUJEAN Benoît: Les coûts du traitement des eaux usées en Wallonie.

COLLIN Denis: Étude préparatoire pour la conception d'un système d'information géographique: guide de l'analyse de l'existant.

DA COL Anne: Photogrammétrie terrestre: mesures expédiées aux levers archéologiques.

DAXHELET Delphine: Géoréférenciation d'une photographie numérisée au sol.

DEFÊCHEREUX Olivier: Étude des zones de loisirs dans les communes des bassins de l'Ourthe et de la Salm.

LAPLANCHE François: Modélisation conceptuelle de projet S.I.G. avec U.M.L.

LEMARCHAL Vincent: Évaluation de la politique des sites d'intérêt régional.

SAINTE Jean Christophe: Prototype de publication sur Internet de la base de données océanographiques IDOD.

SEBA Gautier: Analyse diachronique entre orthophotoplans numériques communaux et images satellite à très haute résolution Ikonos.

\section{2}

option géographie fondamentale

BÉGUÉ Valérie: Les inondations dans le bassin de la Sûre et de la Moselle luxembourgeoise. Bilans hydrologiques de l'Attert et de deux de ses affluents.

CHARLIER Julien: Réflexion radar au sein de la tourbe de remplissage de traces périglaciaires de la fagne des Deux Séries (Hautes Fagnes, Belgique).

COLLARD Sébastien: Contribution à l'étude géomorphologique du bassin de la Chefna et de la vallée de l'Amblève dans les Fonds de Quarreux.

DONNAY François: Détection, analyse et comparaison des vagues de froid hivernales observées en Belgique de 1901 à 1998. Étude menée à partir des séries de températures extrêmes quotidiennes des stations de Denée Maredsous et de Malonne Saint Berthuin et des faits rapportés par la presse.

\section{HANNECART Xavier: La fusion des communes: 25 ans après!}

HOMBROUCKX Nathalie: Analyse hydrologique du bassin de la Gette. Identification et récurrence du débit à pleins bords. Zones inondables et localisation de l'habitat. Transports en solution et en suspension.

HORION Stéphanie: Apport de la télédétection dans l'étude de l'évolution des formations végétales du littoral de Phù Long (île de Cat $\mathrm{Ba}$, Vietnam).

JASCHKO Guillaume: Représentations des modifications territoriales des habitants de Dour. Contribution à la recherche sur les sensibilités territoriales et à la gestion du territoire en fonction des sentiments des habitants envers les lieux.

MOCCIA Teodoro: Retour de la population dans les villes. Le cas des quartiers liégeois.

PIROTTE Benjamin. Les nouvelles technologies de l'information et de la communication. Les besoins spatiaux du secteur de l'informatique. 
STEVENS Catherine: Apport de la télédétection à la recherche archéologique: anciens canaux et structures anciennes du delta du Mékong.

VAN OVERBEKE Marguerite: Réseaux d'innovation technologique et développement régional. Application au cas de la Wallonie.

WOLLSEIFEN Pauline: Le logement, facteur de discrimination socio spatiale dans une ville anglaise.

option géométrologie

BECKS Christophe: L'étude du marché foncier de la commune de Jalhay.

GREVESSE Jonathan: Influence des perturbations ionosphériques, en particulier des orages géomagnétiques, sur les positions précises calculées au moyen du système G.P.S.

LAMALLE Emmanuel: Application du G.P.S. à des mesures topoclimatiques. Étude pilote lors de campagnes itinérantes.

PÂQUE Damien: La problématique des S.I.G. d'entreprise. Architecture et interopérabilité.

SCHENKE Christophe: Comparaison de différents algorithmes et stratégies de fusion d'images satellitaires (capteur Pleiades H.R.).

VANSUYPEENE Éric: Le calibrage des antennes G.P.S.

2003

option géographie fondamentale

COLOT Alexandre: Étude de la localisation des bureaux dans une ville moyenne: Namur, 67 p. +12 cartes.

de MARNEFFE Geneviève: Étude diachronique, à l'aide de la télédétection, de l'évolution du littoral d'une partie du delta du Fleuve Rouge (Viêt-Nam), 109 p. +1 annexe.

DELVAUX Evelyne: Localisation des entreprises Seveso et études de cas au niveau de la localisation, des risques et des rapports avec la population, 147 p. + atlas hors texte.

DELVENNE Yannick: Contribution à l'étude des profils longitudinaux de la Warche entre Robertville et Stavelot, 83 p. +3 annexes.

FARACI Laurent: Contraintes environnementales et formations quaternaires dans la vallée de l'Ourthe inférieure à Embourg et à Angleur, 97 p. + 3 annexes.

FITSCHY Catherine: Modélisation de la distribution spatiale des précipitations en Sicile, 69 p. +2 annexes.

GOCHEL François: Étude géographique d'une voie romaine. La section de Bavay à Tongres, $87 \mathrm{p} .+26$ annexes.

GOFFIN France: Température de l'air et milieu géographique, étude menée à l'aide de campagnes nocturnes itinérantes, 84 p. +10 annexes.

JASPARD Mathieu: Essai de construction d'un indice météorologique de prévention des feux de végétation dans les landes à molinie. Cas de la fagne de Polleur et de la lande de Streupas, 105 p. +6 annexes.

NOIRFALISE Benjamine: Étude de l'impact d'une garnison militaire sur une région. Le cas du Camp Roi Albert (CRA) à Marche-en-Famenne, 74 p. + fig., tabl. et annexes.

PETIT Sophie: Étude de la glace riche en particules minérales présente à la base d'un glacier froid antarctique (Rhone Glacier, Victoria Land), 64 p. +5 annexes. 
RADOSEVIC Grégory: Analyse spatio-temporelle et hiérarchisation de tells en Haute Gezireh, 172 p. +7 annexes.

VANDERHEYDEN Vincent: Dynamique fluviale du Viroin. Contribution à la détermination des zones inondables du bassin, évolution des inondations, morphogénie et transport de la charge de fond, 114 p. +4 annexes.

option géométrologie et géomatique

DAHMOUN Sandra: Adéquation entre le profil d'accessibilité et le profil de mobilité d'un site: de la conception à la réalisation. Étude d'un cas concret: Ans, 78 p. + 1 annexe.

KROONEN Gaëlle: Analyse des erreurs affectant les positions calculées par GPS sur base de mesures de pseudo-distances, 61 p. +3 annexes.

\section{- Université Libre de Bruxelles}

\section{9}

CORNET Anne: Spatialisation des inputs et outputs de l'Université Libre de Bruxelles en 1997.

CRAHAY Antoine: L'intervention des pouvoirs publics dans la rénovation de logements à Bruxelles. Approche géographique.

DELCROIX Paul: Etude géographique de paramètres abiotiques déterminant la zonation intertidale en milieux rocheux. Application au cas du Cap de la Crèche (Boulonnais, Pasde-Calais, France).

METTEWIE Vittorio: Quarante ans de concentrations dans la sidérurgie européenne.Stratégies et politiques industrielles.

SLEEWAEGEN Suzanne: Contribution à l'étude de la glace d'un lac juxtaglaciaire des Dry Valleys, Antarctique.

STRUELENS Vincent: Etude dendroécologique et dendrochimique de deux peuplements de hêtres (Fagus Sylvatica L.) en Forêt de Soignes.

VAN DEN STEEN Isabelle: Analyse de l'évolution de l'occupation du sol depuis l'émergence du tourisme balnéaire à la côte belge.

VANDER PUTTEN Benoit: Transports en commun bruxellois et relations entre quartiers périphériques.

VERBEKE Véronique: Etude de la distribution 3-D des inclusions salines dans les glaces de mer à texture granulaire.

WAYENS Benjamin: Logiques spatiales des enseignes commerciales en Belgique.

2000

CARELS Didier: Contribution d'un système d'information géographique pour l'analyse topographique à grande échelle de la commune d'olne.

CHARLES Julie: Les nouvelles formes d'organisation du monde agricole en Wallonie: succès et limites du modèle français.

CHARLIER Pierre-Hugues: Schéma de fonctionnement de l'économie à l'échelle locale. Etude du cas de La Louvière.

de BORMAN Laurent: L'inscription spatiale des investissements communaux dans l'espace public et les équipements collectifs. 
DELBART Virginie: Evolution de l'emprise spatiale de la ville de Kinshasa .

HIMMER Nicolas: Distribution 3D d'anions majeurs dans la glace marine située sous la plate-forme de glace flottante de Nansen, Antarctique.

HONORE François: Stratégies de localisation du grand commerce soumis à la loi sur les implantations commerciales 1991-1996.

JUGNON Timothée: Synthèse des données concernant la répartition, la biologie et l'économie du thon.

MEDINA LOCKART Pablo: Cartographie de synthèse de la géographie électorale européenne.

MICHA Laurence: Revitalisation résidentielle de la ville de Liège.

PIEL Frédéric: Analyse de la répartition géographique d'Ips typographus dans l'agglomération bruxelloise.

SEURINCK Laurence: Contribution à l'étude dendroécologique du hêtre (Fagus sylvatica L.) en Moyenne Belgique.

VEKEMANS Yann: Evolution des réactions à des crises sectorielles locales en Flandre et en Wallonie. Le cas de régions de tradition textile: Verviers et Courtrai.

ZWINGS Audrey: La revitalisation résidentielle dans le sud de Schaerbeek.

2001

BASTIN Stéphane: Géographie des étrangers haut-statut à Bruxelles.

BEYS Noémie: La rénovation des gares: un outil de revitalisation urbaine?Le cas du quartier de la gare du Midi à Bruxelles.

DELTOUR Perrine: Bathurst: l'axe structurant de la communauté juive de Toronto.

DE PAOLI Sylvie: Le Maërl: distribution - écologie - applications.

DE SCHOUTHEETE DE TERVAERE Laurent: Analyse et comparaison des structures urbaines de Montréal à Bruxelles.

DE SCHUTTER Tom: Géographie de la restructuration des chemins de fer belges depuis 1930.

FEIJT Caroline: Remise à ciel ouvert de la Woluwe: impacts des aménagements sur la qualité biologique du cours d'eau.

GEERAERTS Laurent: Utilisation d'images satellitaires pour la détection des panaches volcaniques.

LAUREYS François: Rapports des clubs sportifs à leur territoire local. L'exemple des clubs francophones de football de division I belge.

LOUTE Guillaume: Approche des migrations intercommunales en Belgique par la modélisation spatiale.

MOULAERT Sabine: L'impact de changements d'occupation du sol sur les inondations: cas du bassin de la Woluwe.

THAYS Carole: Les migrations vers les quartiers en processus de gentrification à Bruxelles.

2002

BOEREBOOM Thierry: Etude comparative des glaces flottantes de deux lacs proglaciaires antarctiques. 
DUBRUILLE D.: Une géographie des mouvements de jeunesse. Analyse des logiques de répartition spatiale en Communauté Française de Belgique.

CASPERS Catherine: Caractéristiques et protection des eaux souterraines en région karstique: études de cas en Haute-Meuse.

CONSTANT Fabrice: Géochimie isotopique du strontium et flux de calcium dans une hêtraie d'Ardenne méridionale.

DEDONDER Virginie: Evaluation de l'importance relative du processus de calcification par les organismes phytoplanctoniques calcifiants.

FODOR Zoltan: Etude de la charge solide et ionique de la Dyle: recherche des causalités.

GODART J.: Contribution de l'analyse géographique à la réduction des zones suspectées d'être minées. Etude de cas, le village de Glinska Poljana en Croatie.

GREGOIRE A.: «Hommes et paysages»: le pays de Peyresq.

MEKIDECHE F.: Analyse historique de la périurbanisation bruxelloise de 1961 à 1991.

NEBBOT Jeremy: Etude hydrologique du Domaine Nysdam.

NIELSEN M.: La mise en place des aménagements structurants à Bruxelles depuis 1830: vers une grille de lecture des villes.

SCHILTZ C.: Structures et espaces du football de haut niveau en Europe.

VANDEBURIE Julien: Vers une géographie mondiale des conflits actuels: Epistémologie, typologie et cartographie.

VANDERPOORTEN Annick: Contribution à l'étude des différents types de glace de lac en Antarctique: le cas du lac Brownworth (Vallée Wright).

VAN KELST J.: Exploitation d'image satellitaire à faible résolution de nuit pour la caractérisation des villes africaines

VERKINDERE Maud: Etude comparative d'étangs urbains et forestiers: variations nycthémérales des principaux paramètres limnologiques.

WERBROUCK A.: Les traces des paysages ruraux à Uccle.

2003

BASSEE Corinne: L'évolution de la localisation du commerce de luxe à Bruxelles entre 1841 et 1999.

DEBECKER Fabienne: Etude de la glace riche en particules minérales présente à la base d'un glacier froid antarctique (Wright Lower Glacier, Victoria Land).

DEROOVER Samuel: Géographie des syndicats et de leurs rapports de force en Belgique de 1950 à 2000.

HANUS Julien: Qualité des eaux courantes du bassin de la Dyle: impact des affluents sur le niveau de pollution hydrologique.

HEUZE Bruno: Contribution à l'étude hydrologique d'un système karstique dans les calcaires dévoniens: le Fond des Vaulx à Marche en Famenne.

LANGENAKEN Quentin: Etude expérimentale des concentrations en impuretés et en gaz à impact climatique dans les glace de congélation. Cas des eaux à 25 et $35 \%$ de salinité.

LENEL Thomas: Une géographie de la noblesse belge. Analyse des logiques de répartitions spatiales en Belgique. 

expansion.

\section{9}

\section{0}

MAURICE Guy: Utilisation de photographies aériennes au 1/10.000 IRC dans le cadre de l'épidémiologie d'Ips typographus sur l'épicéa dans la région des Vosges.

NEUBOURG Grégory: Les vues paysagères numériques. Un outil de gestion du paysage en

PARMENTIER Benoît: Utilisation de l'imagerie satellitaire à très haute résolution dans l'épidémiologie de l'Ips typographus.

PREVEDELLO Cédric: Consommation, accessibilité financière de l'eau de distribution et gestion durable de la ressource dans la région de Charleroi.

ROLLAND Florence: Impacts de la fréquentation humaine sur la population de grands mammifères: l'exemple de la forêt de Soignes.

SCHILTZ Aline: L'émigration portugaise au Grand-Duché de Luxembourg. Analyse de l'impact local dans le village de Fiolhoso.

SOBRIE Jérôme: Etude expérimentale des concentrations en impuretés et en gaz à impact climatique dans les glaces de congélation. Cas des eaux à 0 et $10 \%$ de salinité.

UNGER Jonathan: Vers une géographie de la police locale en Région de Bruxelles-Capitale.

\section{- Vrije Universiteit Brussel}

BAETEN Leen: Typologie van buurten. Een onderzoek in het kader van het VUB-Brussel 2000 project «Crossing Brussels».

CAVELO Manuel: Globalisatie als middel tot ontwikkeling?

DELMELLE Eric: Studie van de mobilitiet in de omgeving van het Leonardkruispunt.

FONTEYN Eva: Fietsen in Brussel. Methodologie om fietsroutes af te bakenen.

PEETERS Helga: Ontbinding van ruimtelijk geaggregeerde eenheden in een categorische datalaag aan de hand van dasymmetrische kartering.

VANDERWALLEN Michel: De herbestemmingvan industriële gebouwen in het Brussels Hoofdstedelijk Gewest.

BRICHAU Veerle: Evolutie van de neerslag in België (Ukkel).

COPPENS Kim: Methoden tot het verhogen van de leefbaarheid in sociale hoogbouw.

DEKNOPPER Roel: Definitie van een geschikte index voor het ontwikkelen van projecties voor wereldkaarten met een minimale oppervlakte- en vormvertekening.

OP DE BEECK Christelle: De problematiek rond achtergestelde buurten in Mechelen.

RIP Kim: Ontwikkeling van een applicatie voor de-aggregatie van ruimtelijke gegevens toegepast op het Brussels Hoofdstedelijk Gewest.

VAN CAMPENHOUT Elise: Urbanisatie en gezondheid in ontwikkelingslanden: Het beleid en de organizatie van de gezondheidszorg Case studies Mumbai (Bombay) en Mexico City.

VANDERHOYDONK Michael: Neerslagrecyclage als parameter voor de wisselwerking tussen het klimaat en de vegetatie in West-Afrika.

75 VERBEIREN Boud: Toepassing van een netwerkanalyse ter bepaling van de mogelijkheden van intermodaal binnenvaart/weg transport in België. 
WEEMAELS Simon: Een onderzoek naar de bewonersparticipatie in de Gentse wijk MuideMeulestede in het kader van het SIF.

2001

ANTHEUNIS Jan: Habitatmodellering van de kerkuil (Tyto alba) in het Hageland.

BIERNAUX Valerie: Erosiemodellering en landinrichting, het stoombekken van de Oued Laou (Rifgebergte, Noord-Marokko).

DE BRABANDER Sang: Radio-echo sounding op sofiyskiy gletsjer, Altaï, Rusland: analyse van interne structuren en basale condities.

DECOSTER Anja: Schatting van het sub-pixel aandeel van bodembedekkingsklassen voor Europa op basis van vgt-gegevens.

DE SMEDT Bert: Numeriek modelleren van de frontvariaties van Sofiyskiy gletsjer, Altaigebergte, Rusland, als respons op het klimaatsignaal.

HEYVAERT Vanessa: Erosiekartering en modellering te Ban Huai Plachon, Tambon Khao Hin Sorn, Amphoe Amphoe Phanomsarakam, Chachoengcao Province Thailand.

MARTIN Geert: Stadsvernieuwing door wijkcontracten in het Brussels Gewest. Een onderzoek over de wijken «Dauw» en «Houwaert-Bossuet».

MONTÉ Mark: De evolutie van het klimaat in Ukkel.

RIP Kim: Ontwikkeling van een amplicatie voor de-aggregatie van ruimtelijke gegevens toegepast op het Brussels Hoofdstedelijk Gewest.

THAENS Karen: Het Wijkontwikkelingsplan Oud-Berchem/Groenenhoek. Een evaluatie na 2 jaar Berchems Model.

VAN DE KEER Lennie: Optimalisatie van continentale kaartprojecties voor gebruik in de kleinschalige atlascartografie.

VERLÉ Wendy: Studie van de energiebalans van Heito Gletsjer, Dronning Maud Land, Oost-Antarctica.

2002

FEYS Inge: Bruselas Portuguesa. Onderzoek over de aanwezigheid van Portugezen en de Portugese cultuur in het Brusselse Hoofdstedelijk Gewest.

MEEUSEN Tieltsje: Ontwikkelingsmogelijkheden van culturele-industrieën in OudMolenbeek: een verkennend onderzoek.

MYNCKE Rafaël: Analyse van de migratiepatronen in Brussel tussen 1992 en 2000.

PEETERS Iris: Actuele en historische erosie in Wortegem-Petegem.

REEKMANS Liesbeth: Classificatie van stedelijk Landgebruik op basis van Zeer Hoge Resolutie Satellietgegevens.

UYTTERSPROT Filip: Gentrificatie, een vergelijkende studie op internationaal vlak: Spitalfields, De Dansaertwijk en De Pijp.

VERDOODT Mark: De veelkleurige stedelijke openbare ruimten: Een onderzoek naar het gebruik van pleinen in Brusselse wijken.

\section{3}

ADRIAENS Juliaan: Lamotsite, Mechelen. Binnenstedelijke heropleving en sociale verdringing: Impactstudie van een grootschalig bouwproject in een regionale stad.

BOULPAEP Koen: Toepassen van de wavelettechnologie in de klimatologie. 
771

\section{NOTES}

1. Cette liste fait suite à celle publiée dans le Bulletin de la Société Belge d'Etudes Géographiques (SOBEG), 1998, 2, pp. 244-272/Deze lijst is een actualisering van deze die in 1988 gepubliceerd werd in het Tijdschrift van de Belgische Vereniging voor Aardrijkskundige Studies (BEVAS), 1998, 2, pp. 244-272. 